\title{
Rationalizability of Choice Functions on General Domains Without Full Transitivity*
}

\author{
WaLter Bossert \\ Département de Sciences Économiques and C.R.D.E. \\ Université de Montréal \\ C.P. 6128, succursale Centre-ville \\ Montréal QC H3C 3J7 \\ Canada \\ FAX: (+1 514) 3437221 \\ e-mail: Walter.Bossert@umontreal.ca \\ Yves Sprumont \\ Département de Sciences Économiques and C.R.D.E. \\ Université de Montréal \\ C.P. 6128, succursale Centre-ville \\ Montréal QC H3C 3J7 \\ Canada \\ FAX: (+1 514) 3437221 \\ e-mail: Yves.Sprumont@umontreal.ca \\ Kotaro Suzumura \\ Institute of Economic Research \\ Hitotsubashi University \\ Kunitachi, Tokyo 186-8603 \\ Japan \\ FAX: (+81 42) 5808353 \\ e-mail: suzumura@ier.hit-u.ac.jp
}

May 2001

* Financial support through grants from the Social Sciences and Humanities Research Council of Canada and a Grant-in-Aid for Scientific Research from the Ministry of Education, Culture, Sports, Science and Technology of Japan is gratefully acknowledged. 


\begin{abstract}
The rationalizability of a choice function by means of a transitive relation has been analyzed thoroughly in the literature. However, not much seems to be known when transitivity is weakened to quasi-transitivity or acyclicity. We describe the logical relationships between the different notions of rationalizability involving, for example, the transitivity, quasi-transitivity, or acyclicity of the rationalizing relation. Furthermore, we discuss sufficient conditions and necessary conditions for rational choice on arbitrary domains. Transitive, quasi-transitive, and acyclical rationalizability are fully characterized for domains that contain all singletons and all two-element subsets of the universal set. Journal of Economic Literature Classification No.: D11.
\end{abstract}

Keywords: Rational Choice, Quasi-Transitivity, Acyclicity, Base Domains. 


\section{Introduction}

The intuitive conception of rational choice as optimizing behavior, irrespective of the nature of the objective that the rational decision maker tries to optimize, has been studied extensively in the literature. Beginning with the revealed preference theory of consumer demand on competitive markets, which is due to Samuelson (1938, 1947 Chapter V, 1948, 1950) and Houthakker (1950), the early phase of the theory of rational choice was devoted to the analysis of choices from budget sets only.

Uzawa (1957) and Arrow (1959) freed this theory from this exclusive concern by introducing the general concept of a choice function defined on the domain of all subsets of a universal set of alternatives. Following this avenue, Sen (1971, 1982), Schwartz (1976), Bandyopadhyay and Sengupta (1991), and many others succeeded in characterizing optimizing choice corresponding to fine demarcations in the degree of consistency of the objective to be maximized. Most notably, the theory of rational choice on such full domains was greatly simplified by the equivalence results between several revealed preference axioms, for example, the weak axiom of revealed preference and the strong axiom of revealed preference, whose subtle difference had been regarded as lying at the heart of the integrability problem for a competitive consumer. However, this simplification was obtained at a price which some may think is much too high. Instead of assuming that the domain of a choice function consists solely of the set of budget sets, it is assumed that "the domain includes all finite subsets of [the universal set of alternatives for choice] whether or not it includes any other subset" (Sen, 1971, 1982 p. 47). It deserves emphasis that "it is not necessary that even all finite sets be included in the domain. All the results and proofs would continue to hold even if the domain includes all pairs and triples but not all finite sets" (Sen, 1971, 1982 pp. 48-49).

Whatever stance one may want to take vis-à-vis Sen's argument in favor of his domain assumption, it is interesting to see what we can make of the concept of a rational choice function irrespective of which assumption we care to specify on its domain, thereby focussing directly on what the logic of rational choice - and nothing else - entails in general. A crucial step along this line was taken by Richter (1966, 1971), Hansson (1968) and Suzumura (1976, 1977, 1983 Chapter 2) who assumed the domain of a choice function to be an arbitrary family of non-empty subsets of an arbitrary nonempty universal set of alternatives without any algebraic or topological structure. These authors succeeded in axiomatizing the concept of a fully rational choice function, that is, a choice function resulting from the optimization of an underlying transitive preference ordering. Yet, 
"cold winds blow through unstructured sets" (Howard, 1971 p. xvii), and there remains a large gap between the theory of rational choice functions with the Arrow-Sen domain and that with the Richter-Hansson domain. In more concrete terms, the Richter-Hansson approach has not yet delivered an axiomatization of rational choice functions where the underlying preference relation is not fully transitive but possesses weaker properties such as quasi-transitivity or acyclicity. The purpose of this paper is to narrow down this gap along two lines.

In the first place, we focus on choice functions defined on what we call base domains, which contain all singletons and pairs of alternatives included in some universal set. On these domains, we provide axiomatizations of choice functions rationalized by preference relations that are not fully transitive. In addition, a new characterization of transitive rational choice is provided for those domains. The concept of a rational choice as an optimizing choice is binary in nature in that the choice from any (possibly very large) set is to be accounted for in terms of a binary relation. In this sense, base domains seem to be the most natural domains to work with in the theory of rational choice. Triples need not be included in a base domain even though consistency properties involving three or more alternatives (namely, quasi-transitivity and acyclicity) are imposed, a feature which distinguishes our approach from the Arrow-Sen framework.

In the second place, we develop new necessary conditions for choice functions defined on arbitrary domains to be rationalized by preference relations that are merely quasitransitive or acyclical. Furthermore, in the acyclical case, we present a new sufficient condition, and we prove that it is weaker than a set of sufficient conditions in the earlier literature.

Within the context of the consistency properties of transitivity, quasi-transitivity, and acyclicity, we also explore the implications of all possible notions of rational choice as an optimizing choice both on arbitrary domains and on base domains. Furthermore, we analyze both maximal-element rationalizability and greatest-element rationalizability (see Sen, 1997, for example).

The remainder of the paper is organized as follows. Section 2 introduces the concept of rationalizability, along with some preliminary observations. Logical relationships are examined in Section 3. Section 4 contains our charcaterization results on base domains. In Section 5 , we present sufficient conditions and necessary conditions on arbitrary domains. Section 6 concludes. 


\section{Rationalizable Choice Functions}

The set of positive (nonnegative) integers is denoted by $\mathbb{N}\left(\mathbb{N}_{0}\right)$. For a set $S,|S|$ is the cardinality of $S$. Let $X$ be a universal nonempty set of alternatives. $\mathcal{X}$ is the power set of $X$ excluding the empty set. A choice function is a mapping $C: \Sigma \rightarrow \mathcal{X}$ such that $C(S) \subseteq S$ for all $S \in \Sigma$, where $\Sigma \subseteq \mathcal{X}$ with $\Sigma \neq \emptyset$ is the domain of $C$. In addition to arbitrary nonempty domains, we consider binary domains which are domains $\Sigma \subseteq \mathcal{X}$ such that $\{S \in \mathcal{X}|| S \mid=2\} \subseteq \Sigma$, and base domains which are domains $\Sigma \subseteq \mathcal{X}$ such that $\{S \in \mathcal{X}|| S \mid=1$ or $|S|=2\} \subseteq \Sigma$.

Let $R \subseteq X \times X$ be a binary relation on $X$. The asymmetric factor $P(R)$ of $R$ is given by $(x, y) \in P(R)$ if and only if $(x, y) \in R$ and $(y, x) \notin R$ for all $x, y \in X$.

A relation $R \subseteq X \times X$ is (i) reflexive if, for all $x \in X,(x, x) \in R$; (ii) complete if, for all $x, y \in X$ such that $x \neq y,(x, y) \in R$ or $(y, x) \in R$; (iii) transitive if, for all $x, y, z \in X$, $[(x, y) \in R$ and $(y, z) \in R]$ implies $(x, z) \in R$; (iv) quasi-transitive if $P(R)$ is transitive; (v) acyclical if, for all $K \in \mathbb{N} \backslash\{1\}$ and for all $x^{0}, \ldots, x^{K} \in X,\left(x^{k-1}, x^{k}\right) \in P(R)$ for all $k \in\{1, \ldots, K\}$ implies $\left(x^{K}, x^{0}\right) \notin P(R)$; (vi) asymmetric if, for all $x, y \in X,(x, y) \in R$ implies $(y, x) \notin R$.

The transitive closure of $R \subseteq X \times X$ is denoted by $\bar{R}$, that is, for all $x, y \in X$, $(x, y) \in \bar{R}$ if there exist $K \in \mathbb{N}$ and $x^{0}, \ldots, x^{K} \in X$ such that $x^{0}=x, x^{K}=y$ and $\left(x^{k-1}, x^{k}\right) \in R$ for all $k \in\{1, \ldots, K\}$. Clearly, $\bar{R}$ is transitive and, because we can set $K=1$, it follows that $R \subseteq \bar{R}$.

The direct revealed preference relation $R_{C} \subseteq X \times X$ of a choice function $C$ with an arbitrary domain $\Sigma$ is defined as follows. For all $x, y \in X,(x, y) \in R_{C}$ if there exists $S \in \Sigma$ such that $x \in C(S)$ and $y \in S$. The (indirect) revealed preference relation of $C$ is the transitive closure $\overline{R_{C}}$ of the direct revealed preference relation $R_{C}$. If $\Sigma$ is a base domain, the base relation $B_{C} \subseteq X \times X$ of $C$ is defined by letting, for all $x, y \in X$, $(x, y) \in B_{C}$ if $x \in C(\{x, y\})$.

For $S \in \Sigma$ and a relation $R \subseteq X \times X$, the set of $R$-greatest elements in $S$ is $G(S, R)=\{x \in S \mid(x, y) \in R$ for all $y \in S\}$, and the set of $R$-maximal elements in $S$ is $M(S, R)=\{x \in S \mid(y, x) \notin P(R)$ for all $y \in S\}$. A choice function $C$ is greatestelement rationalizable if there exists a relation $R$ on $X$ such that $C(S)=G(S, R)$ for all $S \in \Sigma$. $C$ is maximal-element rationalizable if there exists a relation $R$ on $X$ such that $C(S)=M(S, R)$ for all $S \in \Sigma$.

Depending on the properties that we might want to impose on a rationalizing relation, different notions of rationalizability can be defined. In particular, our focus is on 
transitivity, quasi-transitivity, and acyclicity. We refer to those properties as consistency conditions, and we let T,Q,A,R, and C stand for transitivity, quasi-transitivity, acyclicity, reflexivity, and completeness, respectively. Each notion of rationalizability is identified by a list of properties assumed to be satisfied by the rationalizing relation, followed by the type of rationalizability (greatest-element or maximal-element rationalizability). For example, QC-G means greatest-element rationalizability by a quasi-transitive and complete relation, ARC-M is maximal-element rationalizability by an acyclical, reflexive, and complete relation, etc..

We conclude this section with some preliminary results. We first present the following lemma, due to Samuelson $(1938,1948)$; see also Richter (1971). It states that the direct relevaled preference relation must be contained in any greatest-element rationalizing relation.

Lemma 1 If $R$ greatest-element rationalizes $C$, then $R_{C} \subseteq R$.

Proof. Suppose $R$ greatest-element rationalizes $C$ and $(x, y) \in R_{C}$ for some $x, y \in X$. By definition of $R_{C}$, there exists $S \in \Sigma$ such that $x \in C(S)$ and $y \in S$. Because $R$ greatest-element rationalizes $C$, this implies $(x, y) \in R$.

If $R$ is transitive and greatest-element rationalizes $C$, it also follows that the strict preference relation corresponding to $R_{C}$ must be contained in the strict preference relation of $R$, that is, $P\left(R_{C}\right) \subseteq P(R)$ (see Bossert, 2001). On an arbitrary domain, this result is no longer true if transitivity is weakened to quasi-transitivity.

Example 1 Let $X=\{x, y\}, \Sigma=\{\{x, y\}\}$, and $C(\{x, y\})=\{x\}$. The relation $R$ defined by

$$
R=\{(x, x),(x, y),(y, x)\}
$$

is quasi-transitive and greatest-element rationalizes $C$ but we have

$$
P\left(R_{C}\right)=\{(x, y)\} \nsubseteq \emptyset=P(R) .
$$

Even if a greatest-element rationalizing relation $R$ is reflexive and complete, $P\left(R_{C}\right)$ need not be contained in $P(R)$.

Example 2 Let $X=\{x, y, z, w\}, \Sigma=\{\{x, z\},\{x, y, w\}\}$, and define $C(\{x, z\})=\{z\}$ and $C(\{x, y, w\})=\{x, w\}$. The relation $R$ given by

$$
R=(X \times X) \backslash\{(x, z),(y, w)\}
$$


is quasi-transitive, reflexive, and complete and greatest-element rationalizes $C$. We have $(x, y) \in P\left(R_{C}\right)$ and $(x, y) \notin P(R)$ and, hence, $P\left(R_{C}\right) \nsubseteq P(R)$.

The implication discussed above does hold on a base domain even if no consistency requirement such as transitivity, quasi-transitivity or acyclicity is imposed.

Lemma 2 Suppose $\Sigma$ is a base domain. If $R$ greatest-element rationalizes $C$, then $P\left(R_{C}\right) \subseteq P(R)$.

Proof. Suppose $(x, y) \in P\left(R_{C}\right)$ for some $x, y \in X$. This implies $(x, y) \in R_{C}$ and, by Lemma $1,(x, y) \in R$. By way of contradiction, suppose $(y, x) \in R$. Because $\Sigma$ is a base domain, $\{y\} \in \Sigma$. By the nonemptiness of $C(\{y\}), y \in C(\{y\})$. Hence, $(y, y) \in R_{C}$ and, using Lemma 1 again, $(y, y) \in R$. Because $\Sigma$ is a base domain, $\{x, y\} \in \Sigma$. Because $(y, x) \in R,(y, y) \in R$, and $R$ greatest-element rationalizes $C$, we must have $y \in C(\{x, y\})$ and hence $(y, x) \in R_{C}$. But this contradicts the assumption that $(x, y) \in P\left(R_{C}\right)$.

A final preliminary observation concerns an axiom that is necessary for greatestelement rationalizability even without any restrictions on a rationalizing relation. This requirement is referred to as the $\mathrm{V}$-axiom in Richter (1971); we call it D-congruence (D for 'direct revelation') in order to have a systematic terminology throughout this paper.

D-Congruence: For all $S \in \Sigma$, for all $x \in S$, if $(x, y) \in R_{C}$ for all $y \in S$, then $x \in C(S)$. We state Richter's (1971) result that D-congruence is necessary for greatest-element rationalizability by an arbitrary relation on an arbitrary domain. For completeness, we provide a proof.

Lemma 3 If $C$ is greatest-element rationalizable, then $C$ satisfies D-congruence.

Proof. Suppose $R$ greatest-element rationalizes $C$, and let $S \in \Sigma$ and $x \in S$ be such that $(x, y) \in R_{C}$ for all $y \in S$. By Lemma $1,(x, y) \in R$ for all $y \in S$ and, because $R$ greatest-element rationalizes $C$, it follows that $x \in C(S)$.

Richter (1971) shows that D-congruence is not only necessary but also sufficient for greatest-element rationalizability by an arbitrary binary relation on an arbitrary domain. Moreover, the axiom is necessary and sufficient for greatest-element rationalizability by a reflexive (but otherwise unrestricted) relation on an arbitrary domain. The requirement remains, of course, necessary for rationalizability if we restrict attention to base domains. However, if we add a consistency requirement such as transitivity, quasi-transitivity, or acyclicity, D-congruence by itself is not sufficient for rationalizability, even on base domains. 


\section{Logical Relationships}

We provide a full description of the logical relationships between the different notions of rationalizability that can be defined in this setting. The possible definitions of rationalizability that can be obtained depend on which consistency requirement is adopted (namely, transitivity, quasi-transitivity or acyclicity) and on whether reflexivity or completeness are added. Furthermore, a distinction between greatest-element rationalizability and maximal-element rationalizability is made.

We state all logical relationships between the different notions of rationality analyzed in this paper in two theorems - one for arbitrary domains and one for base domains. For convenience, a diagrammatic representation is employed: all axioms that are depicted within the same box are equivalent, and an arrow pointing from one box $b$ to another box $b^{\prime}$ indicates that the axioms in $b$ imply those in $b^{\prime}$, and the converse implication is not true. In addition, of course, all implications resulting from chains of arrows depicted in the diagram are valid.

Theorem 1 Suppose $\Sigma$ is a general domain. Then

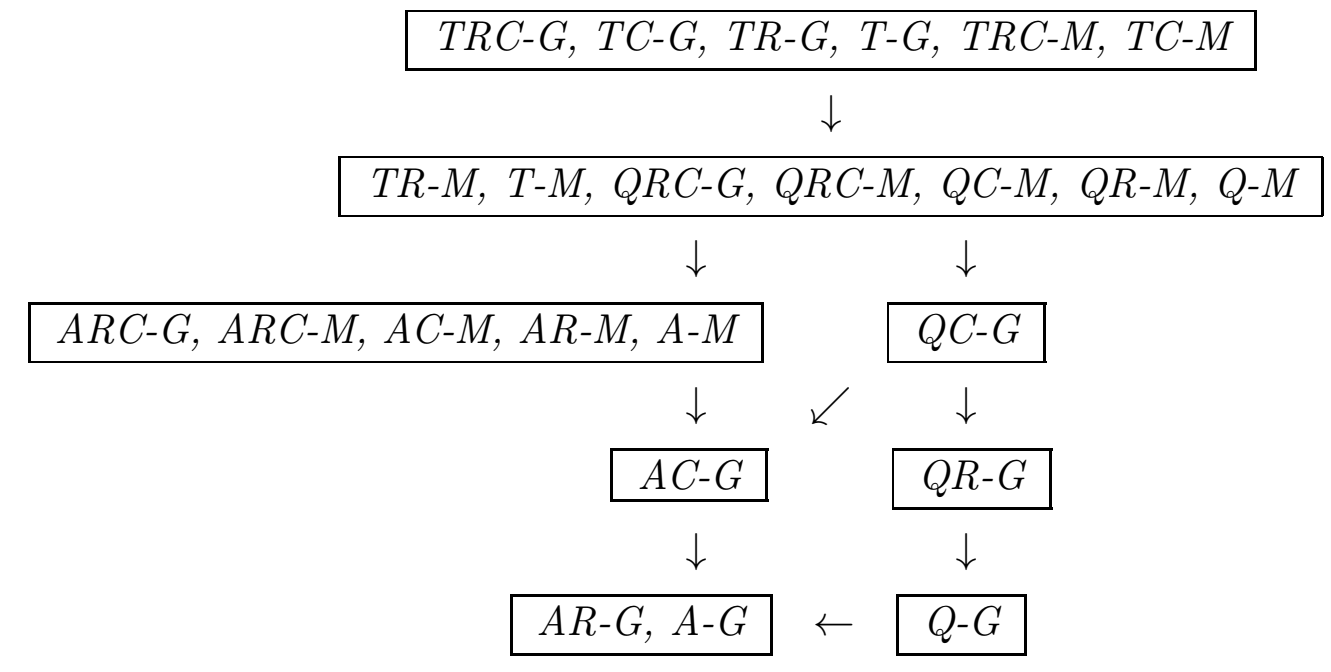

Proof. We proceed as follows. In Step 1, we prove the equivalence of all axioms that appear in the same box. In Step 2, we show that all implications depicted in the theorem statement are valid. In Step 3, we demonstrate that no further implications are true other than those resulting from chains of implications established in Step 2.

Step 1. We prove the equivalence of the axioms for each of the four boxes containing more than one axiom. 
1.a. We first prove the equivalence of the axioms in the top box.

That TRC-G, TC-G, TR-G, and T-G are equivalent follows directly from Richter's $(1966,1971)$ results.

Because maximal elements and greatest elements coincide for a reflexive and complete relation, it follows that TRC-G and TRC-M are equivalent.

Finally, we show that TC-M implies TRC-M. Suppose $R$ is a transitive and complete relation that maximal-element rationalizes $C$, that is, $C(S)=M(S, R)$ for all $S \in \Sigma$. Let

$$
R^{\prime}=R \cup\{(x, x) \mid x \in X\}
$$

It follows immediately that $R^{\prime}$ is reflexive, complete, and transitive. Furthermore, $P\left(R^{\prime}\right)=$ $P(R)$ and, therefore, $M\left(S, R^{\prime}\right)=M(S, R)=C(S)$ for all $S \in \Sigma$, which implies that $R^{\prime}$ maximal-element rationalizes $C$.

1.b. Next, we prove that the axioms in the second box from the top are equivalent.

That TR-M and T-M are equivalent can be shown using the same construction as in the proof of the equivalence of TRC-M and TC-M.

Clearly, QRC-G and QRC-M are equivalent because greatest and maximal elements coincide for a reflexive and complete relation.

Next, we show that Q-M implies QRC-M. Suppose $R$ is a quasi-transitive relation that maximal-element rationalizes $C$. Define $R^{\prime}$ by

$$
R^{\prime}=\{(x, y) \in X \times X \mid(y, x) \notin P(R)\}
$$

Regardless of the properties possessed by $R, R^{\prime}$ is always reflexive and complete and, furthermore, $P\left(R^{\prime}\right)=P(R)$ and hence

$$
G\left(S, R^{\prime}\right)=M\left(S, R^{\prime}\right)=M(S, R) \text { for all } S \in \Sigma
$$

Since $R$ is quasi-transitive and $P\left(R^{\prime}\right)=P(R), R^{\prime}$ is quasi-transitive as well.

That $R^{\prime}$ maximal-element rationalizes $C$ follows immediately from (2) and the assumption that $R$ maximal-element rationalizes $C$.

To complete this part of the proof, it is sufficient to establish the equivalence of T-M and QRC-G.

First, we show that T-M implies QRC-G. Suppose $C$ is maximal-element rationalizable by a transitive relation $R$. Define the relation $R^{\prime}$ as in (1). As in the argument proving the previous implication, $R^{\prime}$ is quasi-transitive, reflexive, and complete, and (2) implies that $R^{\prime}$ greatest-element rationalizes $C$. 
To prove that QRC-G implies T-M, suppose $R$ is a quasi-transitive, reflexive, and complete relation that greatest-element rationalizes $C$. Define

$$
R^{\prime}=P(R)
$$

$R^{\prime}$ is transitive because $R$ is quasi-transitive. Furthermore, we have $P\left(R^{\prime}\right)=P(R)$ and hence

$$
M\left(S, R^{\prime}\right)=M(S, R)=G(S, R) \text { for all } S \in \Sigma,
$$

where the second equality follows from reflexivity and completeness of $R$. Since $R$ greatestelement rationalizes $C$ it follows from (3) that $R^{\prime}$ maximal-element rationalizes $C$.

1.c. We prove that the axioms ARC-G and all axioms involving maximal-element rationalizability by an acyclical relation are equivalent.

Again, the equivalence of ARC-G and ARC-M follows immediately because the greatest and maximal elements of a reflexive and complete relation concide.

Finally, we show that A-M implies ARC-M. Suppose $R$ is an acyclical relation that maximal-element rationalizes $C$. Define $R^{\prime}$ as in (1). Again, it is clear that $R^{\prime}$ is reflexive and complete. Since $R$ is acyclical and $P\left(R^{\prime}\right)=P(R), R^{\prime}$ is acyclical as well. The argument showing that $R^{\prime}$ maximal-element rationalizes $C$ is identical to the one used in 1.b.

1.d. To complete the first part of the proof, it remains to be shown that A-G implies AR-G. Suppose $R$ is acyclical and greatest-element rationalizes $C$. Define

$$
R^{\prime}=(R \cup\{(x, x) \mid x \in X\}) \backslash\{(x, y) \in X \times X \mid(x, x) \notin R \text { and } y \neq x\}
$$

Clearly, $R^{\prime}$ is reflexive. Furthermore, by definition of $R^{\prime}$, we have

$$
\left[(x, x) \notin R \Rightarrow(x, y) \notin R^{\prime}\right] \text { for all } x \in X, \text { for all } y \in X \backslash\{x\} \text {. }
$$

Now suppose $R^{\prime}$ is not acyclical. Then there exist $K \in \mathbb{N} \backslash\{1\}$ and $x^{0}, \ldots, x^{K} \in X$ such that $\left(x^{k-1}, x^{k}\right) \in P\left(R^{\prime}\right)$ for all $k \in\{1, \ldots, K\}$ and $\left(x^{K}, x^{0}\right) \in P\left(R^{\prime}\right)$. Clearly, we can, without loss of generality, assume that the $x^{k}$ are pairwise distinct. By $(5),\left(x^{k-1}, x^{k-1}\right) \in$ $R$ for all $k \in\{1, \ldots, K+1\}$. But this implies that we have $\left(x^{k-1}, x^{k}\right) \in P(R)$ for all $k \in\{1, \ldots, K\}$ and $\left(x^{K}, x^{0}\right) \in P(R)$ by definition of $R^{\prime}$, contradicting the acyclicity of $R$.

We now prove that $R^{\prime}$ greatest-element rationalizes $C$. For future reference, note that the argument used in the proof does not depend on any of the properties of $R$ other than the observation that it greatest-element rationalizes $C$. Let $S \in \Sigma$ and $x \in S$. 
Suppose $x \in C(S)$. Because $R$ greatest-element rationalizes $C$, we have $(x, y) \in R$ for all $y \in S$ which, in particular, implies $(x, x) \in R$. Therefore, by $(4),(x, y) \in R^{\prime}$ for all $y \in S$ and hence $x \in G\left(S, R^{\prime}\right)$.

Now suppose $x \in G\left(S, R^{\prime}\right)$. Therefore, $(x, y) \in R^{\prime}$ for all $y \in S$. If $S=\{x\}, x \in C(S)$ follows from the nonemptiness of $C(S)$. If there exists $y \in S$ such that $y \neq x,(5)$ implies $(x, x) \in R$. Therefore, because $(x, y) \in R^{\prime}$ implies $(x, y) \in R$ for all $y \in S$ and $R$ greatest-element rationalizes $C$, we immediately obtain $x \in C(S)$.

Step 2. The only nontrivial implication is that QC-G implies QR-G. Suppose $R$ is quasi-transitive and complete and greatest-element rationalizes $C$. Define the (reflexive) relation $R^{\prime}$ as in (4). Next, we prove that $R^{\prime}$ is quasi-transitive. Suppose $(x, y) \in P\left(R^{\prime}\right)$ and $(y, z) \in P\left(R^{\prime}\right)$. By $(5),(x, x) \in R$ and $(y, y) \in R$. Suppose $(x, y) \notin P(R)$. Because $R$ is complete, we have $(y, x) \in R$. Because $(y, y) \in R$, it follows that $(y, x) \in R^{\prime}$ by definition of $R^{\prime}$, contradicting $(x, y) \in P\left(R^{\prime}\right)$. Therefore, $(x, y) \in P(R)$.

We now distinguish two cases.

Case a. $(z, z) \in R$. Analogously to the above proof demonstrating that $(x, y) \in$ $P(R)$, we obtain $(y, z) \in P(R)$ in this case. Because $R$ is quasi-transitive, it follows that $(x, z) \in P(R)$. Because $(x, x) \in R$ and $(x, z) \in P(R)$, we must have $(x, z) \in R^{\prime}$ by definition of $R^{\prime}$. Furthermore, $(z, x) \notin R$ implies $(z, x) \notin R^{\prime}$ by definition of $R^{\prime}$ and, consequently, we obtain $(x, z) \in P\left(R^{\prime}\right)$.

Case b. $(z, z) \notin R$. By $(5)$, we obtain $(z, x) \notin R^{\prime}$. Suppose $(x, z) \notin R^{\prime}$. Because $(x, x) \in R$, this implies $(x, z) \notin R$ by definition of $R^{\prime}$ and hence $(z, x) \in P(R)$ by the completeness of $R$. Because $R$ is quasi-transitive, we obtain $(z, y) \in P(R)$ and hence $(y, z) \notin R$. Because $(z, z) \notin R$, the definition of $R^{\prime}$ implies $(y, z) \notin R^{\prime}$, contradicting $(y, z) \in P\left(R^{\prime}\right)$. Therefore, $(x, z) \in R^{\prime}$ and, because $(z, x) \notin R^{\prime}$, it follows that $(x, z) \in$ $P\left(R^{\prime}\right)$.

That $R^{\prime}$ greatest-element rationalizes $C$ can be shown using the same proof as in 1.d.

Step 3. To prove that no further implications other than those resulting from Step 2 are valid, it is sufficient to provide examples showing that (a) QRC-G does not imply T-G; (b) QC-G does not imply ARC-G; (c) ARC-G does not imply Q-G; (d) QR-G does not imply AC-G; and (e) Q-G does not imply QR-G.

3.a. QRC-G does not imply T-G.

Example 3 Let $X=\{x, y, z\}$ and $\Sigma=\mathcal{X} \backslash\{\{x, y, z\}\}$. Define the choice function $C$ by letting $C(\{t\})=\{t\}$ for all $t \in X, C(\{x, y\})=\{x, y\}, C(\{x, z\})=\{z\}$, and $C(\{y, z\})=\{y, z\}$. This choice function is greatest-element rationalizable by the quasi- 
transitive, reflexive, and complete relation

$$
R=(X \times X) \backslash\{(x, z)\}
$$

Suppose $C$ is greatest-element rationalizable by a transitive relation $R^{\prime}$. Because $x \in$ $C(\{x, y\})$, we must have $(x, x) \in R^{\prime}$ and $(x, y) \in R^{\prime}$. Analogously, $y \in C(\{y, z\})$ implies $(y, z) \in R^{\prime}$. By the transitivity of $R^{\prime}$, it follows that $(x, z) \in R^{\prime}$ and, together with $(x, x) \in R^{\prime}, x \in C(\{x, z\})$, contradicting the definition of $C$.

3.b. QC-G does not imply ARC-G.

Example 4 Let $X=\{x, y, z, w\}$ and $\Sigma=\{\{x, w\},\{y, z\},\{y, w\},\{x, y, w\},\{y, z, w\}\}$, and define $C(\{x, w\})=\{w\}, C(\{y, z\})=\{y\}, C(\{y, w\})=\{y, w\}, C(\{x, y, w\})=\{w\}$, and $C(\{y, z, w\})=\{y\}$. This choice function is greatest-element rationalized by the quasitransitive and complete relation $R$ given by

$$
\{(x, y),(x, z),(x, w),(y, y),(y, z),(y, w),(z, x),(z, y),(z, w),(w, x),(w, y),(w, w)\} .
$$

Suppose $R^{\prime}$ is acyclical, reflexive, and complete and greatest-element rationalizes $C$.

Because $C(\{y, z\})=\{y\}$ and $R^{\prime}$ is reflexive, we obtain $(y, z) \in P\left(R^{\prime}\right)$. Analogously, because $C(\{x, w\})=\{w\}$ and $R^{\prime}$ is reflexive, we must have $(w, x) \in P\left(R^{\prime}\right)$.

Because $y \in C(\{y, w\})$ and $y \notin C(\{x, y, w\})$, we must have $(y, x) \notin R^{\prime}$ and, because $R^{\prime}$ is complete, it follows that $(x, y) \in P\left(R^{\prime}\right)$. Analogously, because $w \in C(\{y, w\})$ and $w \notin C(\{y, z, w\})$, we must have $(w, z) \notin R^{\prime}$ and, because $R^{\prime}$ is complete, it follows that $(z, w) \in P\left(R^{\prime}\right)$.

Therefore, we have established that $(x, y) \in P\left(R^{\prime}\right),(y, z) \in P\left(R^{\prime}\right),(z, w) \in P\left(R^{\prime}\right)$, and $(w, x) \in P\left(R^{\prime}\right)$, contradicting the acyclicity of $R^{\prime}$.

3.c. ARC-G does not imply Q-G.

Example 5 Let $X=\{x, y, z\}$ and $\Sigma=\mathcal{X} \backslash\{\{x, y, z\}\}$. Define the choice function $C$ by letting $C(\{t\})=\{t\}$ for all $t \in X, C(\{x, y\})=\{x\}, C(\{x, z\})=\{x, z\}$, and $C(\{y, z\})=\{y\}$. This choice function is greatest-element rationalizable by the acyclical, reflexive, and complete relation

$$
R=\{(x, x),(x, y),(x, z),(y, y),(y, z),(z, x),(z, z)\}
$$

Suppose $C$ is greatest-element rationalizable by a quasi-transitive relation $R^{\prime}$. Because $y \in C(\{y, z\})$, we have $(y, y) \in R^{\prime}$. Therefore, $y \notin C(\{x, y\})$ implies $(x, y) \in P\left(R^{\prime}\right)$. 
Analogously, $z \in C(\{x, z\})$ implies $(z, z) \in R^{\prime}$ and, therefore, $z \notin C(\{y, z\})$ implies $(y, z) \in P\left(R^{\prime}\right)$. Because $R^{\prime}$ is quasi-transitive, it follows that $(x, z) \in P\left(R^{\prime}\right)$ and hence $(z, x) \notin R^{\prime}$. Because $R^{\prime}$ greatest-element rationalizes $C$, this implies $z \notin C(\{x, z\})$, contradicting the definition of $C$.

3.d. QR-G does not imply AC-G.

Example 6 Let $X=\{x, y, z\}$ and $\Sigma=\{\{x, y\},\{x, z\},\{x, y, z\}\}$. Define the choice function $C$ by letting $C(\{x, y\})=\{x, y\}, C(\{x, z\})=\{x, z\}$, and $C(\{x, y, z\})=\{x\} . C$ is greatest-element rationalizable by the quasi-transitive and reflexive relation

$$
R=\{(x, x),(x, y),(x, z),(y, x),(y, y),(z, x),(z, z)\},
$$

but it cannot be greatest-element rationalized by a complete relation. By way of contradiction, suppose $R^{\prime}$ is such a relation. By completeness, we must have

$$
(y, z) \in R^{\prime}
$$

or

$$
(z, y) \in R^{\prime} .
$$

Suppose (6) is true. Because $R^{\prime}$ greatest-element rationalizes $C$ and $y \in C(\{x, y\})$, it follows that $(y, x) \in R^{\prime}$ and $(y, y) \in R^{\prime}$. Together with (6) and the greatest-element rationalizability of $C$ by $R^{\prime}$, we obtain $y \in C(\{x, y, z\})$, contradicting the definition of $C$.

Now suppose (7) is true. Because $R^{\prime}$ greatest-element rationalizes $C$ and $z \in C(\{x, z\})$, it follows that $(z, x) \in R^{\prime}$ and $(z, z) \in R^{\prime}$. Together with (7) and the greatest-element rationalizability of $C$ by $R^{\prime}$, we obtain $z \in C(\{x, y, z\})$, contradicting the definition of $C$.

3.e. Q-G does not imply QR-G.

Example 7 Let $X=\{x, y, z, w\}$ and $\Sigma=\{\{x, y\},\{y, z\},\{z, w\},\{x, z, w\}\}$, and define the choice function $C$ by letting $C(\{x, y\})=\{y\}, C(\{y, z\})=\{z\}, C(\{z, w\})=\{z, w\}$, and $C(\{x, z, w\})=\{w\}$. This choice function is greatest-element rationalized by the quasi-transitive relation $R$ given by

$$
\{(x, y),(y, x),(y, y),(z, y),(z, z),(z, w),(w, x),(w, z),(w, w)\} .
$$

Suppose $R^{\prime}$ is quasi-transitive and reflexive and greatest-element rationalizes $C$. By reflexivity, $(x, x) \in R^{\prime}$ and, because $x \notin C(\{x, y\})$, we must have $(y, x) \in P\left(R^{\prime}\right)$. Because 
$y \in C(\{x, y\})$, it follows that $(y, y) \in R^{\prime}$ and, hence, $y \notin C(\{y, z\})$ implies $(z, y) \in P\left(R^{\prime}\right)$. By quasi-transitivity, we obtain $(z, x) \in P\left(R^{\prime}\right)$.

Because $z \in C(\{z, w\})$, it follows that $(z, z) \in R^{\prime}$ and $(z, w) \in R^{\prime}$. Together with $(z, x) \in P\left(R^{\prime}\right)$ and the assumption that $R^{\prime}$ greatest-element rationalizes $C$, we obtain $z \in C(\{x, z, w\})$, which contradicts the definition of $C$.

Regarding the implications presented in the above theorem, it is worth pointing out some surprising differences between those notions of rationalizability encompassing transitivity and those that merely require quasi-transitivity or acyclicity. Most strikingly, as soon as we weaken full transitivity to quasi-transitivity, not even reflexivity is implied as a property of a greatest-element rationalizing relation. On the other hand, all notions of maximal-element rationalizability coincide if merely quasi-transitivity rather than full transitivity is required.

The results regarding the logical relationships between our rationalizability axioms simplify dramatically when base domains are considered. The presence of all one-element and two-element sets in $\Sigma$ guarantees that every greatest-element rationalizing relation must be reflexive and complete and, as a consequence, all rationality requirements involving greatest-element rationalizability with a given consistency requirement become equivalent. All implications of Theorem 1 are preserved and, other than those just mentioned, there are no additional ones. Those demanding transitivity are stronger than those where merely quasi-transitivity is required which, in turn, imply (but are not implied by) all axioms where the rationalizing relation is acyclical. These observations are summarized in the following theorem.

Theorem 2 Suppose $\Sigma$ is a base domain. Then

\begin{tabular}{c}
$\frac{1}{T R C-G, T C-G, T R-G, T-G, T R C-M, T C-M}$ \\
$\downarrow$ \\
\hline$T R-M, T-M, Q R C-G, Q C-G, Q R-G, Q-G, Q R C-M, Q C-M, Q R-M, Q-M$ \\
$\downarrow$ \\
$A R C-G, A C-G, A R-G, A-G, A R C-M, A C-M, A R-M, A-M$ \\
\hline
\end{tabular}

Proof. We divide the proof into the same three steps as in Theorem 1.

Step 1. We prove the equivalence of the axioms for each of the three boxes. 
1.a. The equivalence of the axioms in the top box follows from Theorem 1.

1.b. Now consider the axioms in the second box. Given Theorem 1, it is sufficient to prove that Q-G implies QRC-G. Suppose $R$ is a quasi-transitive relation that rationalizes $C$. Because $\Sigma$ is a base domain, $\{x\} \in \Sigma$ for all $x \in X$. Because $C(S)$ is nonempty for all $S \in \Sigma$, it follows that $(x, x) \in R_{C}$ for all $x \in X$ and, by Lemma $1,(x, x) \in R$ for all $x \in X$. Hence $R$ is reflexive. Analogously, because $\Sigma$ is a base domain, $\{x, y\} \in \Sigma$ for all $x, y \in X$ such that $x \neq y$, and the nonemptiness of $C(S)$ for all $S \in \Sigma$ implies $(x, y) \in R_{C}$ or $(y, x) \in R_{C}$ for all $x, y \in X$ such that $x \neq y$. Using Lemma 1 again, it follows that $R$ is complete. Thus, QRC-G is satisfied.

1.c. That the axioms in the last box are equivalent follows from Theorem 1 and the assumption that $\Sigma$ is a base domain as in 1.b.

Step 2. The implications in the theorem statement are trivial.

Step 3. To prove that no further implications are valid, Examples 3 and 5 can be employed.

As shown in Theorem 2, there are only three different versions of rationalizability for base domains. As a consequence, we can restrict attention to the rationalizability axioms TRC-G, QRC-G, and ARC-G in this case, keeping in mind that, by Theorem 2, all other rationalizability requirements discussed in this paper are covered as well by our results.

Note that, in the case of transitive greatest-element rationalizability, all definitions of rationalizability are equivalent even if $\Sigma$ only contains all two-element sets but not necessarily the singletons; this is a consequence of the observation that if $R$ is a transitive (and complete) relation greatest-element rationalizing $C$, it is always possible to find a reflexive and transitive (and complete) relation that contains $R$ and rationalizes $C$ as well; see Richter (1966, 1971). Therefore, the equivalence of all axioms involving a transitive greatest-element rationalization can be established for binary domains as well. We do not state the corresponding result formally as a separate theorem because our focus is on quasi-transitive and acyclical rationalizability in this paper.

\section{Characterizations for Binary and Base Domains}

If we restrict attention to base domains (that is, domains $\Sigma$ that contain all one-element and two-element sets), the analysis of quasi-transitive and acyclical rationalizability is significantly less complex than in the case of an arbitrary domain, and we obtain 'clean' characterization results. In addition, we formulate a new characterization result regarding transitive rationalizability for binary domains. The full power of a base domain is not 
required in the transitive case because reflexivity can always be added as a property of a rationalizing relation as long as transitivity is satisfied.

Note that the assumption of having a base domain differs in an important aspect from the assumption used by Sen (1971), which stipulates that not only the sets of cardinality two, but also those of cardinality three are in $\Sigma$. (Sen did not require the singletons to be in the domain due to the observation that, for transitive greatest-element rationalizability, reflexivity can always be added as a property of a greatest-element rationalizing relationsee the discussion at the end of the previous section.) It is interesting to note that, in order to obtain useful and applicable results for quasi-transitive and acyclical rationalizing relations, those sets of cardinality three are not required in the domain, even though these consistency properties impose restrictions on three or more alternatives which may be distinct. It is not sufficient to assume that we have a binary domain (that is, a domain containing all two-element sets). The singleton sets are needed if transitivity is weakened to quasi-transitivity or acyclicity because, without full transitivity, reflexivity of a rationalizing relation can no longer be guaranteed. Base domains have also been used by Herzberger (1973) but he did not pursue the same questions we address in this paper.

\subsection{Transitive Rationalizability}

In the case of binary domains, we obtain a new characterization of TRC-G that employs a weaker axiom than Richter's (1966) congruence axiom to be defined in Section 5. This axiom-which we call T-congruence-is defined as follows.

T-Congruence: For all $x, y, z \in X$, for all $S \in \Sigma$, if $(x, y) \in R_{C},(y, z) \in R_{C}, x \in S$ and $z \in C(S)$, then $x \in C(S)$.

Note that, in contrast to congruence, T-congruence does not apply to chains of direct revealed preference of an arbitrary length but merely to chains involving three elements. For binary domains, T-congruence is necessary and sufficient for TRC-G. Of course, Tcongruence is necessary for greatest-element rationalizability by a transitive relation on an arbitrary domain but it is not sufficient unless specific domain assumptions are made.

Example 8 Let $X=\{x, y, z, w\}, \Sigma=\{\{x, y\},\{y, z\},\{z, w\},\{x, w\}\}$, and define $C$ by $C(\{x, y\})=\{x\}, C(\{y, z\})=\{y\}), C(\{z, w\})=\{z\}$, and $C(\{x, w\})=\{w\}$. This choice function satisfies T-congruence but it is not greatest-element rationalizable by a transitive relation. To see this, suppose $R$ is transitive and greatest-element rationalizes $C$. The definition of $C$ yields $(x, y) \in R_{C},(y, z) \in R_{C},(z, w) \in R_{C}$, and $(x, x) \in R_{C}$. By Lemma 
1, $(x, y) \in R,(y, z) \in R,(z, w) \in R$, and $(x, x) \in R$. Because $R$ is transitive, we must have $(x, w) \in R$. By definition of greatest-element rationalizability, it follows that $x \in C(\{x, w\})$, a contradiction.

It is an interesting observation that binary domains are sufficient to obtain results of that nature involving transitivity, even though those domains do not necessarily contain all triples. This is in contrast to Sen's (1971) results which crucially depend on having all triples available in the domain. We obtain

Theorem 3 Suppose $\Sigma$ is a binary domain. $C$ satisfies $T R C-G$ if and only if $C$ satisfies T-congruence.

Proof. Let $\Sigma$ be a binary domain. This implies that $R_{C}$ is complete - see the proof of Theorem 2.

Step 1. That TRC-G implies T-congruence follows immediately from Richter's (1966) result and the observation that T-congruence is weaker than his congruence axiom.

Step 2. We show that T-congruence implies TRC-G. Let

$$
R=R_{C} \cup\{(x, x) \mid x \in X\} .
$$

Clearly, $R$ is reflexive by definition, and $R$ is complete because $R_{C}$ is complete. Next, we prove that $R$ is transitive. Suppose $(x, y) \in R$ and $(y, z) \in R$ for some $x, y, z \in X$. If $x=z,(x, z) \in R$ follows from the reflexivity of $R$. If $x \neq z$, it follows that $\{x, z\} \in \Sigma$ because $\Sigma$ is a binary domain. By T-congruence, $x \in C(\{x, z\})$ and hence $(x, z) \in R_{C}$ which, by Lemma 1 , implies $(x, z) \in R$.

Finally, we show that $R$ greatest-element rationalizes $C$. Let $S \in \Sigma$ and $x \in S$.

Suppose $x \in C(S)$. This implies $(x, y) \in R_{C}$ for all $y \in S$ and hence $(x, y) \in R$ for all $y \in S$. Hence, $x \in G(S, R)$.

Now suppose $x \in G(S, R)$, that is, $(x, y) \in R$ for all $y \in S$. If $S=\{x\}$, we have $C(S)=\{x\}$ because $C(S)$ is nonempty and hence $(x, x) \in R_{C}$. If there exists $y \in S$ such that $y \neq x$, it follows that $(x, y) \in R_{C}$ and, by definition of $R_{C}, x$ must be chosen for some feasible set in $\Sigma$. Thus, again, $(x, x) \in R_{C}$. Therefore, $(x, y) \in R_{C}$ for all $y \in S$. Let $z \in C(S)$. This implies $(z, z) \in R_{C}$. Because $z \in S,(x, z) \in R_{C}$. Letting $y=z$ in the definition of T-congruence, the axiom implies $x \in C(S)$.

Clearly, if $\Sigma$ is a base domain rather than merely a binary domain, $R_{C}$ is reflexive and can be used as the rationalizing relation in the above theorem. 


\subsection{Quasi-Transitive Rationalizability}

To obtain a set of necessary and sufficient conditions for QRC-G in the case of a base domain, we add the following Q-congruence axiom to the D-congruence axiom introduced in Section 2.

Q-Congruence: For all $x, y, z \in X$, for all $S \in \Sigma$, if $(x, y) \in P\left(R_{C}\right),(y, z) \in P\left(R_{C}\right)$ and $x \in S$, then $z \notin C(S)$.

Together with D-congruence, Q-congruence guarantees that the direct revealed preference relation $R_{C}$ is quasi-transitive. Note, again, that we do not need to impose a restriction regarding chains of (strict) revealed preferences of arbitrary length. We obtain

Theorem 4 Suppose $\Sigma$ is a base domain. $C$ satisfies $Q R C-G$ if and only if $C$ satisfies $D$-congruence and Q-congruence.

Proof. Let $\Sigma$ be a base domain. Therefore, $R_{C}$ is reflexive and complete- see the proof of Theorem 2.

Step 1. We first show that QRC-G implies Q-congruence (that D-congruence is implied follows from Lemma 3). Suppose $R$ is a quasi-transitive relation that greatestelement rationalizes $C$. Let $x, y, z \in X$ and $S \in \Sigma$ be such that $(x, y) \in P\left(R_{C}\right),(y, z) \in$ $P\left(R_{C}\right)$, and $x \in S$. By Lemma $2,(x, y) \in P(R)$ and $(y, z) \in P(R)$ and, because $R$ is quasi-transitive, $(x, z) \in P(R)$. This implies $(z, x) \notin R$ and because $R$ greatest-element rationalizes $C$, we have $z \notin C(S)$.

Step 2. We show that D-congruence and Q-congruence together imply QRC-G. First, we prove that $R_{C}$ is quasi-transitive. Suppose $(x, y) \in P\left(R_{C}\right)$ and $(y, z) \in P\left(R_{C}\right)$ for some $x, y, z \in X$. Because $\Sigma$ is a base domain, $\{x, z\} \in \Sigma$. By Q-congruence, $z \notin C(\{x, z\})$ and hence $x \in C(\{x, z\})$ which implies $(x, z) \in R_{C}$. Since $R$ is reflexive, $(z, z) \in R_{C}$. If $(z, x) \in R_{C}$, D-congruence implies $z \in C(\{x, z\})$, a contradiction. Therefore, $(x, z) \in$ $P\left(R_{C}\right)$.

The rest of the proof proceeds as in Richter (1971) by showing that $R_{C}$ greatest-element rationalizes $C$, given D-congruence. Let $S \in \Sigma$ and $x \in S$. Suppose $x \in C(S)$. This implies $(x, y) \in R_{C}$ for all $y \in S$ and hence $x \in G\left(S, R_{C}\right)$. Now suppose $x \in G\left(S, R_{C}\right)$, that is, $(x, y) \in R_{C}$ for all $y \in S$. By D-congruence, $x \in C(S)$.

D-congruence and Q-congruence are independent on base domains, as shown by means of the following examples.

Example 9 Let $X=\{x, y, z\}$ and $\Sigma=\mathcal{X} \backslash\{\{x, y, z\}\}$, and define $C(\{t\})=\{t\}$ for all 
$t \in X, C(\{x, y\})=\{x\}, C(\{y, z\})=\{y\}$, and $C(\{x, z\})=\{z\}$. This choice function satisfies $D$-congruence but violates $Q$-congruence.

Example 10 Let $X=\{x, y, z\}$ and $\Sigma=\mathcal{X}$, and define $C(\{t\})=\{t\}$ for all $t \in X$, $C(\{x, y\})=\{x, y\}, C(\{y, z\})=\{y, z\}, C(\{x, z\})=\{x, z\}$, and $C(\{x, y, z\})=\{y, z\}$. This choice function satisfies $Q$-congruence but violates $D$-congruence.

Q-congruence is a weaker axiom than the quasi-transitivity of $R_{C}$; it is only in conjunction with D-congruence that it implies that the revealed preference relation is quasitransitive. Strengthening Q-congruence to the quasi-transitivity of $R_{C}$ does not allow us to drop D-congruence in the above characterization. Note that the above example showing that Q-congruence does not imply D-congruence is such that $R_{C}$ is quasi-transitive, and recall that D-congruence is necessary for greatest-element rationalizability on any domain (Lemma 3).

Given that we employ a base domain, it is natural to ask whether the base relation $B_{C}$ could be used in place of the revealed preference relation $R_{C}$ in the formulation of D-congruence and Q-congruence. This is not the case.

Example 11 Let $X=\{x, y, x\}$ and $\Sigma=\mathcal{X}$, and define $C(\{t\})=\{t\}$ for all $t \in X$, $C(\{x, y\})=\{y\}, C(\{x, z\})=\{z\}, C(\{y, z\})=\{y, z\}$, and $C(\{x, y, z\})=\{x, y, z\}$. This choice function satisfies the modifications of D-congruence and $Q$-congruence where $R_{C}$ is replaced with $B_{C}$ but it does not satisfy D-congruence (and, thus, fails to be greatestelement rationalizable by any binary relation). Note that replacing $R_{C}$ with $B_{C}$ leads to a weakening of D-congruence but to a strengthening of $Q$-congruence.

\subsection{Acyclical Rationalizability}

If quasi-transitivity is weakened to acyclicity, it seems natural to replace Q-congruence by the following A-congruence axiom in order to obtain a characterization of the respective rationalizability property on a base domain.

A-Congruence: For all $x, y \in X$, for all $S \in \Sigma$, if $(x, y) \in \overline{P\left(R_{C}\right)}, x \in S$ and $y \in C(S)$, then $x \in C(S)$.

It is indeed the case that D-congruence and A-congruence together are necessary and sufficient for ARC-G on base domains. However, A-congruence by itself is stronger than the acyclicity of $R_{C}$ and, thus, a stronger characterization result can be obtained by employing acyclicity instead of A-congruence. 
Theorem 5 Suppose $\Sigma$ is a base domain. $C$ satisfies $A R C-G$ if and only if $C$ satisfies D-congruence and $R_{C}$ is acyclical.

Proof. Let $\Sigma$ be a base domain. Again, it follows that $R_{C}$ is reflexive and complete.

Step 1. We first show that ARC-G implies that $R_{C}$ is acyclical (again, that Dcongruence is implied follows from Lemma 3).

Suppose $R$ is an acyclical, reflexive, and complete relation that greatest-element rationalizes $C$. Let $K \in \mathbb{N} \backslash\{1\}$ and $x^{0}, \ldots, x^{K} \in X$ be such that $\left(x^{k-1}, x^{k}\right) \in P\left(R_{C}\right)$ for all $k \in\{1, \ldots, K\}$. By Lemma $2,\left(x^{k-1}, x^{k}\right) \in P(R)$ for all $k \in\{1, \ldots, K\}$. Because $R$ is acyclical, we have $\left(x^{K}, x^{0}\right) \notin P(R)$ and, since $R$ is complete, $\left(x^{0}, x^{K}\right) \in R$. Because $R$ is reflexive, $\left(x^{K}, x^{K}\right) \in R$. Because $\Sigma$ is a base domain, $\left\{x^{0}, x^{K}\right\} \in \Sigma$. Because $R$ greatest-element rationalizes $C, x^{0} \in C\left(\left\{x^{0}, x^{K}\right\}\right)$ and hence $\left(x^{0}, x^{K}\right) \in R_{C}$, which implies $\left(x^{K}, x^{0}\right) \notin P\left(R_{C}\right)$.

Step 2. D-congruence and the acyclicity of $R_{C}$ together imply ARC-G because Dcongruence implies that $R_{C}$ greatest-element rationalizes $C$, as was shown in the last paragraph of the proof of Theorem 4.

That D-congruence and the acyclicity of $R_{C}$ are independent is shown by Examples 9 and 10. Analogously, D-congruence cannot be replaced with an axiom that merely applies to the base relation $B_{C}$ instead of $R_{C}$.

\section{Conditions for Arbitrary Domains}

In this section, we examine greatest-element rationalizability and maximal-element rationalizability on completely arbitrary domains under various assumptions regarding the properties of a rationalizing relation. For each of the consistency conditions quasitransitivity and acyclicity, we examine new necessary conditions and sufficient conditions for rational choice. In order to put our new results in perspective, we begin with a statement of Richter's (1996) well-known result characterizing TRC-G for arbitrary domains.

\subsection{Transitive Rationalizability}

Richter (1966) shows that the congruence axiom is necessary and sufficient for TRC-G. Congruence is defined as follows.

Congruence: For all $x, y \in X$, for all $S \in \Sigma$, if $(x, y) \in \overline{R_{C}}, y \in C(S)$ and $x \in S$, then $x \in C(S)$. 
We obtain

Theorem 6 C satisfies TRC-G if and only if $C$ satisfies congruence.

Proof. See Richter (1966).

An alternative condition that is equivalent to TRC-G is Houthakker's axiom of revealed preference; see, for example, Suzumura (1983, p. 48).

\subsection{Quasi-Transitive Rationalizability}

If we move from a base domain to an arbitrary domain, the conjunction of Q-congruence and D-congruence ceases to be sufficient for QRC-G, as can be seen from Example 4. Moreover, Q-congruence is not a necessary condition for QRC-G either, as demonstrated by the following example.

Example 12 Let $X=\{x, y, z, u, v, w\}, \Sigma=\{\{x, y, u\},\{x, z, w\},\{y, z, v\}\}$, and define $C(\{x, y, u\})=\{x, u\}, C(\{x, z, w\})=\{z, w\}$, and $C(\{y, z, v\})=\{y, v\}$. This choice function is greatest-element rationalizable by the quasi-transitive, reflexive, and complete relation $R$ given by

$$
\begin{gathered}
\{(x, t) \mid t \in X \backslash\{w\}\} \cup\{(y, t) \mid t \in X \backslash\{u\}\} \cup\{(z, t) \mid t \in X \backslash\{v\}\} \\
\cup\{(u, t) \mid t \in X\} \cup\{(v, t) \mid t \in X\} \cup\{(w, t) \mid t \in X\}
\end{gathered}
$$

Since $(x, y) \in P\left(R_{C}\right)$ and $(y, z) \in P\left(R_{C}\right)$, Q-congruence requires $z \notin C(\{x, z, w\})$, contradicting the definition of $C$.

The formulation of necessary and sufficient conditions for greatest-element or maximalelement rationalizability by a quasi-transitive relation is a complex task. We will return to this issue at the end of this subsection. It is not too difficult to find plausible sufficient conditions and necessary conditions, though. We begin by stating a known result presenting a sufficient condition for QRC-G (and, thus, all rationality requirements involving rationalizability by a quasi-transitive relation).

To do so, we define the relation $E_{C}$ by letting, for all $x, y \in X,(x, y) \in E_{C}$ if

there exists $S \in \Sigma$ such that $x \in C(S)$ and $y \in S \backslash C(S)$.

This relation has been used by Suzumura (1976). The following axiom turns out to be sufficient for QRC-G. 
Strong Axiom of Revealed Preference: For all $x, y \in X$, for all $S \in \Sigma$, if $(x, y) \in \overline{E_{C}}$ and $x \in S$, then $y \notin C(S)$.

Note that, by definition, $E_{C}$ contains $P\left(R_{C}\right)$ and, consequently, the strong axiom of revealed preference is stronger than Q-congruence.

We obtain

Theorem 7 If $C$ satisfies the strong axiom of revealed preference, then $C$ satisfies $Q R C$ $G$. The converse implication is not true.

Proof. See Suzumura (1983, p. 50).

A condition that is equivalent to the strong axiom of revealed preference (and, thus, an alternative sufficient condition for QRC-G) is Uzawa's axiom of revealed preference; see, for example, Suzumura (1983, p. 48).

Now we present a condition that is necessary but not sufficient for QRC-G, even if combined with D-congruence. This axiom involves a recursive construction. The idea is to identify circumstances that force a strict preference between two elements of $X$ and impose a condition ensuring that this forced strict preference is transitive, as required by the quasi-transitivity of a rationalizing relation.

Suppose $C$ is greatest-element rationalizable by a quasi-transitive, reflexive, and complete relation $R$. Consider a feasible set $S \in \Sigma$ and distinct elements $x, y \in S$ such that $y$ is not chosen in $S$ but $y$ is directly revealed preferred to all $z \in S \backslash\{x, y\}$. By Lemma $1,(y, z) \in R$ for all $z \in S \backslash\{x, y\}$ and, together with the reflexivity and completeness of $R, y \in S \backslash C(S)$ requires that $x$ be declared strictly preferred to $y$ according to $R$ and, by quasi-transitivity, all chains of strict preference thus established must be respected as well. Moreover, once it is implied that $x$ is declared strictly preferred to $y$ according to the above argument (or, more generally, according to the transitive closure of the relation thus obtained), this strict preference may have further implications: there may exist another set $T \in \Sigma$ such that $x, y \in T, x$ is declared preferred to all $z \in T \backslash\{x, y, w\}$ for some $w \in S \backslash\{x, y\}$, and $x$ is not chosen in $T$. In that case, we must declare a strict preference for $w$ over $x$ according to $R$. This procedure can be repeated recursively, and we now present a formal definition of this recursion, followed by a necessary condition for QRC-G based on this recursive construction. This recursion is analogous to the one employed in Bossert (2001).

Define the relation $F_{C}^{0}$ on $X$ as follows. For all $x, y \in X,(x, y) \in F_{C}^{0}$ if

there exists $S \in \Sigma$ such that $x \in S \backslash\{y\}, y \in S \backslash C(S)$, and $(y, z) \in R_{C}$ for all $z \in S \backslash\{x, y\}$. 
Now let $i \in \mathbb{N}$. Let $J_{C}^{i-1}=R_{C} \cup \overline{F_{C}^{i-1}}$, and define the relation $F_{C}^{i}$ on $X$ as follows. For all $x, y \in X,(x, y) \in F_{C}^{i}$ if

$$
\begin{aligned}
{\left[(x, y) \in \overline{F_{C}^{i-1}}\right] \quad \text { or } \quad } & \text { there exists } S \in \Sigma \text { such that } x \in S \backslash\{y\}, y \in S \backslash C(S), \\
& \text { and } \left.(y, z) \in J_{C}^{i-1} \text { for all } z \in S \backslash\{x, y\}\right] .
\end{aligned}
$$

Finally, let $J_{C}^{\infty}=\cup_{i \in \mathbb{N}_{0}} J_{C}^{i}$ and $F_{C}^{\infty}=\cup_{i \in \mathbb{N}_{0}} \overline{F_{C}^{i}}$. The following axiom turns out to be necessary for QRC-G.

Recursive Q-Congruence: For all $x, y \in X$, if $(x, y) \in F_{C}^{\infty}$, then $(y, x) \notin J_{C}^{\infty}$.

We obtain

Theorem 8 If $C$ satisfies $Q R C$ - $G$, then $C$ satisfies recursive $Q$-congruence. The converse implication is not true.

Proof. Suppose $R$ is a quasi-transitive, reflexive, and complete relation that greatestelement rationalizes $C$. We first prove that

$$
F_{C}^{\infty} \subseteq P(R)
$$

Clearly, by definition of $F_{C}^{\infty}$, it is sufficient to prove that $\overline{F_{C}^{i}} \subseteq P(R)$ for all $i \in \mathbb{N}_{0}$. We proceed by induction.

Step 1. $i=0$. We first show that $F_{C}^{0} \subseteq P(R)$. Suppose $(x, y) \in F_{C}^{0}$ for some $x, y \in X$. By definition, there exists $S \in \Sigma$ such that $(y, z) \in R_{C}$ for all $z \in S \backslash\{x, y\}, x \in S \backslash\{y\}$, and $y \in S \backslash C(S)$. By Lemma $1,(y, z) \in R$ for all $z \in S \backslash\{x, y\}$ and, because $R$ is reflexive, $(y, y) \in R$. Because $y \in S \backslash C(S)$ and $R$ greatest-element rationalizes $C$, we must have $(y, x) \notin R$. Hence, because $R$ is complete, $(x, y) \in P(R)$.

Because $R$ is quasi-transitive (that is, $P(R)$ is transitive) and $\overline{F_{C}^{0}}$ is the transitive closure of $F_{C}^{0}$, it follows that $\overline{F_{C}^{0}} \subseteq P(R)$.

Step 2. Let $i \in \mathbb{N}$ and suppose $\overline{F_{C}^{j}} \subseteq P(R)$ for all $j \in\{0, \ldots, i-1\}$. Let $(x, y) \in F_{C}^{i}$ for some $x, y \in X$. By definition, there are two cases.

2.a. $(x, y) \in \overline{F_{C}^{i-1}}$. In this case, $(x, y) \in P(R)$ follows from the induction hypothesis.

2.b. There exists $S \in \Sigma$ such that $(y, z) \in J_{C}^{i-1}$ for all $z \in S \backslash\{x, y\}, x \in S \backslash\{y\}$, and $y \in S \backslash C(S)$. Let $z \in S \backslash\{x, y\}$. By definition of $J_{C}^{i-1}$ we have $(y, z) \in R_{C}$ or $(y, z) \in \overline{F_{C}^{i-1}}$. If $(y, z) \in R_{C}$, Lemma 1 implies $(y, z) \in R$. If $(y, z) \in \overline{F_{C}^{i-1}},(y, z) \in R$ follows from the induction hypothesis. Therefore, $(y, z) \in R$ for all $z \in S \backslash\{x, y\}$ and, using the same argument as in Step 1, we obtain $F_{C}^{i} \subseteq P(R)$ and, by the quasi-transitivity of $R, \overline{F_{C}^{i}} \subseteq P(R)$. This completes the proof of (8). 
Next, we prove that

$$
J_{C}^{\infty} \subseteq R .
$$

Again, it is sufficient to prove that $J_{C}^{i} \subseteq R$ for all $i \in \mathbb{N}_{0}$. Let $(x, y) \in J_{C}^{i}$ for some $x, y \in X$. By definition, $(x, y) \in R_{C}$ or $(x, y) \in \overline{F_{C}^{i}}$. If $(x, y) \in R_{C},(x, y) \in R$ follows from Lemma 1. If $(x, y) \in \overline{F_{C}^{i}},(x, y) \in R$ follows from the proof of (8). Therefore, (9) is true.

To complete the proof that QRC-G implies recursive Q-congruence, we proceed by contradiction. Suppose recursive Q-congruence is violated. Then there exists $x, y \in X$ such that $(x, y) \in F_{C}^{\infty}$ and $(y, x) \in J_{C}^{\infty}$. By (8) and (9), we have $(x, y) \in P(R)$ and $(y, x) \in R$, a contradiction.

To see that the converse implication is not true, consider the following example.

Example 13 Let $X=\{x, y, z, u, v, w\}$ and $\Sigma=\{\{x, y, u\},\{y, z, v\},\{x, z, w\}\}$ and define $C$ by letting $C(\{x, y, u\})=\{x\}, C(\{y, z, v\})=\{y\}$, and $C(\{x, z, w\})=\{z\}$. It is straightforward to check that $F_{C}^{0}=\emptyset$. It follows that $J_{C}^{0}=R_{C}, J_{C}^{i}=J_{C}^{0}=R_{C}$ for all $i \in \mathbb{N}$, and $F_{C}^{\infty}=F_{C}^{i}=F_{C}^{0}=\emptyset$ for all $i \in \mathbb{I N}$. Thus, recursive Q-congruence is trivially satisfied. Because we will use this example in the following subsection as well, we show that $C$ cannot be greatest-element rationalized by an acyclical, reflexive, and complete relation (and, thus, it cannot be greatest-element rationalized by a quasi-transitive, reflexive, and complete relation). Suppose, by way of contradiction, that $R$ is acyclical, reflexive, and complete and greatest-element rationalizes $C$. Because $y, u \in\{x, y, u\} \backslash C(\{x, y, u\})$ and $R$ is reflexive and complete, we must have

$$
\text { 1.a. }(x, y) \in P(R) \text { or 1.b. }[(x, u) \in P(R) \text { and }(u, y) \in P(R)] \text {. }
$$

Analogously, because $z, v \in\{y, z, v\} \backslash C(\{y, z, v\})$ and $x, w \in\{x, z, w\} \backslash C(\{x, z, w\})$, we have

$$
\text { 2.a. }(y, z) \in P(R) \text { or 2.b. }[(y, v) \in P(R) \text { and }(v, z) \in P(R)]
$$

and

$$
\text { 3.a. }(z, x) \in P(R) \text { or 3.b. }[(z, w) \in P(R) \text { and }(w, x) \in P(R)] \text {. }
$$

If 1.a, 2.a, and 3.a are true, we immediately obtain a contradiction to the acyclicity of $R$.

If 1.a, 2.a, and 3.b are true, we have $(x, y) \in P(R),(y, z) \in P(R),(z, w) \in P(R)$, and $(w, x) \in P(R)$, contradicting the acyclicity of $R$. Becuase of the symmetric role played by $x, y$, and $z$, analogous contradictions are obtained whenever statements i.a, j.a, and k.b are true for any distinct values of $i, j, k \in\{1,2,3\}$. 
If 1.a, 2.b, and 3.b are true, we obtain $(x, y) \in P(R),(y, v) \in P(R),(v, z) \in P(R)$, $(z, w) \in P(R)$, and $(w, x) \in P(R)$, again a violation of acyclicity. Using the symmetric role of $x, y$, and $z$ again, analogous contradictions are obtained whenever statements i.a, $j . b$, and $k . b$ are true for any distinct values of $i, j, k \in\{1,2,3\}$.

Finally, if 1.b, 2.b, and 2.c are true, we obtain $(x, u) \in P(R),(u, y) \in P(R),(y, v) \in$ $P(R),(v, z) \in P(R),(z, w) \in P(R)$, and $(w, x) \in P(R)$, and acyclicity is violated again.

We noted in Lemma 3 that D-congruence is a necessary condition for greatest-element rationalizability by any relation. This raises the question whether recursive Q-congruence implies D-congruence. To see that this is indeed the case, suppose D-congruence is violated. Then there exist $S \in \Sigma$ and $x \in S$ such that $(x, y) \in R_{C}$ for all $y \in S$ and $x \notin C(S)$. By definition, this implies $(y, x) \in F_{C}^{0} \subseteq F_{C}^{\infty}$ for all $y \in S \backslash\{x\}$. Because $C(S) \subseteq S$ is nonempty and $x \in S \backslash C(S), S \backslash\{x\}$ is nonempty. Consider any $y \in S \backslash\{x\}$. Because $(x, y) \in R_{C}$ we have $(x, y) \in J_{C}^{\infty}$. Therefore, we obtain $(y, x) \in F_{C}^{\infty}$ and $(x, y) \in J_{C}^{\infty}$, contradicting recursive Q-congruence.

Furthermore, recursive Q-congruence and Q-congruence are independent. The choice function in Example 12 satisfies recursive Q-congruence (by Theorem 8; note that it satisfies QRC-G) but violates Q-congruence. Conversely, the choice function in Example 4 satisfies Q-congruence but violates recursive Q-congruence. To see this, note first that $R_{C}=\{(y, y),(y, z),(y, w),(w, x),(w, y),(w, w)\}$. In view of the definition of $C$, it follows that $F_{C}^{0}=\{(x, y),(y, z),(z, w),(w, x)\}$, and the transitive closure of $F_{C}^{0}$ is therefore given by $\overline{F_{C}^{0}}=X \times X$. It follows that $F_{C}^{\infty}=J_{C}^{\infty}=X \times X$, a contradiction to recursive Q-congruence.

Because no particular assumptions are formulated regarding the domain of the choice function, it seems that conditions that are both necessary and sufficient cannot be formulated without invoking existential clauses. Moreover, contrary to the transitive case, quasi-transitivity of a greatest-element rationalizing relation does not imply that the asymmetric factor of the revealed preference relation must be contained in this rationalizing relation - see the discussion regarding Lemma 2 in Section 2. These observations appear to be an important part of the reason why there does not exist much literature on the subject of quasi-transitive rational choice on arbitrary domains. Rather than constructing the relation $F_{C}^{\infty}$ one pair of alternatives at a time, a tighter necessary condition would be to establish the existence of an alternative $x \in S$ such that $x$ can be declared 
better than $y$ if $y$ is feasible but not chosen in $S$. This kind of condition involves an existential clause, and conditions of that nature are difficult to verify in practice and, therefore, are of limited interest.

\subsection{Acyclical Rationalizability}

A-congruence (and, thus, the acyclicity of $R_{C}$ ) fails to be sufficient for ARC-G even in the presence of D-congruence, as Example 4 shows. Moreover, the acyclicity of $R_{C}$ (and, thus, A-congruence) is not necessary for ARC-G in the case of a general domain. This is established by Example 12; note that, in Example 12, we have $(x, y) \in P\left(R_{C}\right)$, $(y, z) \in P\left(R_{C}\right)$, and $(z, x) \in P\left(R_{C}\right)$.

As is the case for quasi-transitivity, the formulation of necessary and sufficient conditions for acyclical rationalizability appears to necessitate the use of axioms involving existential clauses. We provide a discussion analogous to the one for quasi-transitivity to illustrate the issues involved. First, we present a new sufficient condition for ARC-G.

Strong A-Congruence: For all $x, y \in X$, for all $S \in \Sigma$, if $(x, y) \in \overline{E_{C}} \cup R_{C}, x \in S$ and $y \in C(S)$, then $x \in C(S)$.

We obtain

Theorem 9 If $C$ satisfies strong A-congruence, then $C$ satisfies $A R C$ - $G$. The converse implication is not true.

Proof. Suppose $C$ satisfies strong A-congruence. First, we prove that $\overline{E_{C}}$ is asymmetric. Suppose, by way of contradiction, that there exist $x, y \in X$ such that $(x, y) \in \overline{E_{C}}$ and $(y, x) \in \overline{E_{C}}$. Therefore, there exist $K, K^{\prime} \in \mathbb{N}, x^{0}, \ldots, x^{K} \in X$, and $z^{0}, \ldots, z^{K^{\prime}} \in X$ such that $x^{0}=z^{K^{\prime}}=x, x^{K}=z^{0}=y,\left(x^{k-1}, x^{k}\right) \in E_{C}$ for all $k \in\{1, \ldots, K\}$, and $\left(z^{k-1}, z^{k}\right) \in E_{C}$ for all $k \in\left\{1, \ldots, K^{\prime}\right\}$. Hence, $\left(x, z^{K^{\prime}-1}\right) \in \overline{E_{C}}$ and $\left(z^{K^{\prime}-1}, x\right) \in E_{C}$. By definition of $E_{C}$, there exists $S \in \Sigma$ such that $z^{K^{\prime}-1} \in C(S)$ and $x \in S \backslash C(S)$, contradicting strong A-congruence.

Now define

$$
R=\left\{(x, y) \in X \times X \mid(y, x) \notin \overline{E_{C}}\right\} \cup R_{C} .
$$

Clearly, $R$ is reflexive and complete by the asymmetry of $\overline{E_{C}}$. To prove that $R$ is acyclical, we first derive the asymmetric factor of $R$. By definition, $(x, y) \in P(R)$ if

$$
\left[(y, x) \notin \overline{E_{C}} \text { or }(x, y) \in R_{C}\right] \text { and }\left[(x, y) \in \overline{E_{C}} \text { and }(y, x) \notin R_{C}\right]
$$


which is equivalent to

$$
\begin{gathered}
{\left[(y, x) \notin \overline{E_{C}} \text { and }(x, y) \in \overline{E_{C}} \text { and }(y, x) \notin R_{C}\right] \text { or }} \\
{\left[(x, y) \in R_{C} \text { and }(x, y) \in \overline{E_{C}} \text { and }(y, x) \notin R_{C}\right]}
\end{gathered}
$$

Using the asymmetry of $\overline{E_{C}}$, this is equivalent to

$$
\left[(x, y) \in \overline{E_{C}} \text { and }(y, x) \notin R_{C}\right] \text { or }\left[(x, y) \in \overline{E_{C}} \text { and }(x, y) \in P\left(R_{C}\right)\right]
$$

or, equivalently,

$$
\left[(x, y) \in \overline{E_{C}} \text { and }(y, x) \notin R_{C}\right]
$$

Now we establish the acyclicity of $R$. Suppose there exist $K \in \mathbb{N} \backslash\{1\}$ and $x^{0}, \ldots, x^{K} \in$ $X$ such that $x^{0}=x, x^{K}=y,\left(x^{k-1}, x^{k}\right) \in \overline{E_{C}}$ and $\left(x^{k}, x^{k-1}\right) \notin R_{C}$ for all $k \in\{1, \ldots, K\}$. Because $\overline{E_{C}}$ is transitive, we have $(x, y) \in \overline{E_{C}}$ and, by the asymmetry of $\overline{E_{C}},(y, x) \notin \overline{E_{C}}$. By (10), this implies $(y, x) \notin P(R)$.

Finally, we show that $R$ greatest-element rationalizes $C$. Let $S \in \Sigma$ and $x \in S$.

Suppose first that $x \in C(S)$. This implies $(x, y) \in R_{C}$ for all $y \in S$ and hence $(x, y) \in R$ for all $y \in S$, and we have $x \in G(S, R)$.

Now suppose $x \in G(S, R)$. This implies

$$
\left[(y, x) \notin \overline{E_{C}} \text { or }(x, y) \in R_{C}\right] \text { for all } y \in S \text {. }
$$

Let $z \in C(S) \subseteq S$. This implies $(z, x) \in R_{C}$. Because $z \in S,(11)$ implies

$$
(z, x) \notin \overline{E_{C}}
$$

or

$$
(x, z) \in R_{C} .
$$

If (12) is true, we must have $x \in C(S)$ because otherwise $(z, x) \in E_{C}$ by definition and hence $(z, x) \in \overline{E_{C}}$.

If (13) is true, $x \in C(S)$ follows from strong A-congruence.

To show that strong A-congruence is not implied by ARC-G, note that Example 12 can be employed here as well: QRC-G (and, thus, ARC-G) is satisfied but we have $(x, z) \in \overline{E_{C}}$ and $z \in C(\{x, z, w\})$ and $x \in\{x, z, w\} \backslash C(\{x, z, w\})$, contradicting strong A-congruence.

Strong A-congruence is a tighter sufficient condition for ARC-G than the set of sufficient conditions established in Suzumura (1983, p. 51). The conditions used in this earlier contribution are the following. 
Weak Axiom of Revealed Preference: For all $x, y \in X$, for all $S \in \Sigma$, if $(x, y) \in E_{C}$ and $x \in S$, then $y \notin C(S)$.

No $E_{C}$-Cycles: For all $x, y \in X$, for all $S \in \Sigma$, if $(x, y) \in \overline{E_{C}}, x \in S$ and $y \in C(S)$, then $x \in C(S)$.

We obtain

Theorem 10 If $C$ satisfies the weak axiom of revealed preference and no $E_{C}$-cycles, then $C$ satisfies strong A-congruence. The converse implication is not true.

Proof. Suppose strong A-congruence is violated. Then there exist $x, y \in X$ such that $(x, y) \in \overline{E_{C}} \cup R_{C}$ and $(y, x) \in E_{C}$. If the weak axiom of revealed preference is satisfied, it follows that $(x, y) \notin R_{C}$. Therefore, we must have $(x, y) \in \overline{E_{C}}$, and we obtain an $E_{C}$-cycle.

To see that the converse implication is not true, consider the following example.

Example 14 Let $X=\{x, y, z\}$ and $\Sigma=\{\{x, y\},\{x, y, z\}\}$ and define $C$ by letting $C(\{x, y\})=\{x, y\}$ and $C(\{x, y, z\})=\{x\}$. It is straightforward to verify that $C$ satisfies strong A-congruence but violates the weak axiom of revealed preference.

To obtain a necessary condition for ARC-G, we employ a recursive construction as in the previous subsection. Let $H_{C}^{0}=F_{C}^{0}$ and, for $i \in \mathbb{N}$, let $L_{C}^{i-1}=R_{C} \cup \overline{H_{C}^{i-1}}$ and define the relation $H_{C}^{i}$ as follows. For all $x, y \in X,(x, y) \in H_{C}^{i}$ if

$$
\begin{aligned}
{\left[(x, y) \in H_{C}^{i-1}\right] \quad \text { or } \quad } & {[\text { there exists } S \in \Sigma \text { such that } x \in S \backslash\{y\}, y \in S \backslash C(S),} \\
& \text { and } \left.(y, z) \in L_{C}^{i-1} \text { for all } z \in S \backslash\{x, y\}\right] .
\end{aligned}
$$

Furthermore, let $L_{C}^{\infty}=\cup_{i \in \mathbb{N}_{0}} L_{C}^{i}$ and $H_{C}^{\infty}=\cup_{i \in \mathbb{N}_{0}} H_{C}^{i}$.

Analogously to recursive Q-congruence, the following condition is necessary for ARCG.

Recursive A-Congruence: For all $x, y \in X$, if $(x, y) \in H_{C}^{\infty}$, then $(y, x) \notin L_{C}^{\infty}$.

We now obtain

Theorem 11 If $C$ satisfies $A R C$ - $G$, then $C$ satisfies recursive $A$-congruence. The converse implication is not true. 
Proof. Suppose $R$ is an acyclical, reflexive, and complete relation that greatest-element rationalizes $C$. To demonstrate that A-congruence is implied, we first prove

$$
H_{C}^{\infty} \subseteq P(R)
$$

Again, by definition of $H_{C}^{\infty}$, it is sufficient to prove that $H_{C}^{i} \subseteq P(R)$ for all $i \in \mathbb{N}_{0}$. We proceed by induction.

Step 1. $i=0$. Because $H_{C}^{0}=F_{C}^{0}$, Step 1 of the proof of Theorem 8 can be employed to conclude that $H_{C}^{0} \subseteq P(R)$.

Step 2. Let $i \in \mathbb{N}$ and suppose $H_{C}^{j} \subseteq P(R)$ for all $j \in\{0, \ldots, i-1\}$. Let $(x, y) \in H_{C}^{i}$ for some $x, y \in X$. By definition, there are two cases.

2.a. $(x, y) \in H_{C}^{i-1}$. In this case, $(x, y) \in P(R)$ follows from the induction hypothesis.

2.b. There exists $S \in \Sigma$ such that $(y, z) \in L_{C}^{i-1}$ for all $z \in S \backslash\{x, y\}, x \in S \backslash\{y\}$, and $y \in S \backslash C(S)$. Let $z \in S \backslash\{x, y\}$. By definition of $L_{C}^{i-1}$ we have $(y, z) \in R_{C}$ or $(y, z) \in \overline{H_{C}^{i-1}}$. If $(y, z) \in R_{C}$, Lemma 1 implies $(y, z) \in R$. If $(y, z) \in \overline{H_{C}^{i-1}}$, there exist $K \in \mathbb{N}$ and $x^{0}, \ldots, x^{K} \in X$ such that $x^{0}=y, x^{K}=z$ and $\left(x^{k-1}, x^{k}\right) \in H_{C}^{i-1}$ for all $k \in\{1, \ldots, K\}$. By the induction hypothesis, $\left(x^{k-1}, x^{k}\right) \in P(R)$ for all $k \in\{1, \ldots, K\}$, and the acyclicity and the completeness of $R$ together imply $(y, z) \in R$. Therefore, $(y, z) \in R$ for all $z \in S \backslash\{x, y\}$ and, using the same argument as in Step 1 of the proof of Theorem 8, we obtain $H_{C}^{i} \subseteq P(R)$. This completes the proof of (14).

Furthermore, we have

$$
L_{C}^{\infty} \subseteq R
$$

the proof of this claim is analogous to the proof of (9).

To complete the proof that recursive A-congruence is satisfied, the same argument as in the proof of Theorem 8 can be employed, where $F_{C}^{\infty}$ and $J_{C}^{\infty}$ are replaced with $H_{C}^{\infty}$ and $L_{C}^{\infty}$, and (8) and (9) are replaced with (14) and (15).

To see that the converse implication is not true, note that the choice function in Example 13 satisfies recursive A-congruence (trivially because $H_{C}^{\infty}=\emptyset$ ) but it cannot be greatest-element rationalized by an acyclical relation.

Recursive A-congruence implies D-congruence; the proof is analogous to the proof establishing that recursive Q-congruence implies D-congruence.

Furthermore, recursive A-congruence does not imply the acyclicity of $R_{C}$ (and, thus, fails to imply A-congruence). This is shown by Example 13. Conversely, recursive Acongruence is not implied by A-congruence (and, thus, it is not implied by the acyclicity of $R_{C}$; Example 4 establishes this claim. 


\section{Concluding Remarks}

We conclude the paper with a brief discussion of some open problems. As mentioned in the text, it seems very difficult to obtain useful necessary and sufficient conditions for QRC-G or for ARC-G on general domains. Given that very indirect implications of preference maximization have to be taken into consideration, the nature of the problem suggests that existential clauses have to be invoked in order to arrive at full characterizations. See also Bossert (2001) for analogous difficulties in a different framework.

If the formulation of clean necessary and sufficient conditions for QRC-G and for ARC-G turns out to be too complex a task, the following more modest objective might be an issue to be addressed in future work. Note that the strong axiom of revealed preference, a sufficient condition for QRC-G, is not implied by TRC-G and, analogously, strong A-congruence is not implied by QRC-G. Likewise, recursive Q-congruence does not imply ARC-G. One direction in which the results of this paper could be extended is to find a condition that is intermediate in strength between TRC-G and QRC-G, and a condition that is implied by QRC-G and implies ARC-G in order to obtain tighter bounds on possible characterizations.

We conclude by remarking that the strong axiom of revealed preference cannot be weakened to strong A-congruence to get a tighter sufficient condition for QRC-G (this is established by Example 5). Similarly, recursive A-congruence cannot be strengthened to recursive Q-congruence to get a tighter necessary condition for ARC-G (again, this is established by Example 5).

\section{References}

Aizerman, M. And F Aleskerov (1995), Theory of Choice, North-Holland, Amsterdam.

Arrow, K.J. (1959), "Rational choice functions and orderings," Economica 26, 121-127. Bandyopadhyay, T. And K. Sengupta (1991), "Revealed preference axioms for rational choice," Economic Journal 101, 202-213.

Bossert, W. (2001), "Choices, consequences, and rationality," Synthese, forthcoming. Hansson, B. (1968), "Choice structures and preference relations," Synthese 18, 443-458. Herzberger, H. (1973), "Ordinal preference and rational choice," Econometrica 41, $187-237$. 
Houthakker, H.S. (1950), "Revealed preference and the utility function," Economica 17, 159-174.

Howard, N. (1971), Paradoxes of Rationality: Theory of Metagames and Political Behavior, MIT Press, Cambridge.

RichteR, M.K. (1966), "Revealed preference theory," Econometrica 34, 635-645.

Richter, M.K. (1971), "Rational choice," in J. Chipman, L. Hurwicz, M. Richter, And H. Sonnenschein (eds.), Preferences, Utility, and Demand, Harcourt Brace Jovanovich, New York, pp. 29-58.

SAmuelson, P.A. (1938), "A note on the pure theory of consumer's behaviour," Economica $5,61-71$.

Samuelson, P.A. (1947), Foundations of Economic Analysis, Harvard University Press, Cambridge.

SAmuelson, P.A. (1948), "Consumption theory in terms of revealed preference," Economica 15, 243-253.

SAmuelson, P.A. (1950), "The problem of integrability in utility theory," Economica 17, 355-385.

Schwartz, T. (1976), "Choice functions, "rationality" conditions, and variations on the weak axiom of revealed preference," Journal of Economic Theory 13, 414-427.

SEn, A.K. (1971), "Choice functions and revealed preference," Review of Economic Studies 38, 307-317.

SEn, A.K. (1982), Choice, Welfare and Measurement, Basil Blackwell, Oxford.

Sen, A.K. (1997), "Maximization and the act of choice," Econometrica 65, 745-779.

Suzumura, K. (1976), "Rational choice and revealed preference," Review of Economic Studies 43, 149-158.

Suzumura, K. (1977), "Houthakker's axiom in the theory of rational choice," Journal of Economic Theory 14, 284-290.

Suzumura, K. (1983), Rational Choice, Collective Decisions and Social Welfare, Cambridge University Press, New York.

Uzawa, H. (1957), "Note on preference and axioms of choice," Annals of the Institute of Statistical Mathematics 8, 35-40. 